\title{
The balance between GMD and OFUT1 regulates Notch signaling pathway activity by modulating Notch stability
}

\author{
Alvaro Glavic*, Ana López-Varea and José F. de Celis \\ Centro de Biología Molecular Severo Ochoa, CSIC and Universidad Autónoma de Madrid, Cantoblanco 28049, Madrid, Spain. \\ *Center for Genomics of the Cell, Faculty of Sciences, Universidad de Chile, Casilla 653, Santiago, Chile.
}

\section{SUMMARY}

The Notch signaling pathway plays an important role in development and physiology. In Drosophila, Notch is activated by its Delta or Serrate ligands, depending in part on the sugar modifications present in its extracellular domain. O-fucosyltransferase-1 (OFUT1) performs the first glycosylation step in this process, O-fucosylating various EGF repeats at the Notch extracellular domain. Besides its O-fucosyltransferase activity, OFUT1 also behaves as a chaperone during Notch synthesis and is able to down regulate Notch by enhancing its endocytosis and degradation. We have reevaluated the roles that O-fucosylation and the synthesis of GDP-fucose play in the regulation of Notch protein stability. Using mutants and the UAS/Gal4 system, we modified in developing tissues the amount of GDP-mannosedeshydratase (GMD), the first enzyme in the synthesis of GDP-fucose. Our results show that GMD activity, and likely the levels of GDPfucose and O-fucosylation, are essential to stabilize the Notch protein. Notch degradation observed under low GMD expression is absolutely dependent on OFUT1 and this is also observed in Notch Abruptex mutants, which have mutations in some potential O-fucosylated EGF domains. We propose that the GDP-fucose/OFUT1 balance determines the ability of OFUT1 to endocytose and degrade Notch in a manner that is independent of the residues affected by Abruptex mutations in Notch EGF domains.

Running title: GMD/OFUT1 balance regulates Notch stability. Key words: Notch, GMD, O-fucosyltranserase, Drosophila.

\section{INTRODUCTION}

The Notch signaling pathway is present from nematodes to humans and carries out fundamental roles in a wide variety of developmental processes (Lai, 2004). In Drosophila, the intercellular communication mediated by this pathway is performed by the Notch receptor $(\mathrm{N})$ and its ligands Delta (Dl) and Serrate (Ser) ligands. Binding of the ligands to Notch induces its proteolytic cleavage within the juxtamembrane domain, leading to the release of the intracellular domain $\left(\mathrm{N}^{\mathrm{ICD}}\right) . \mathrm{N}^{\mathrm{ICD}}$ translocates to the nucleus where it binds to the Suppressor of Hairless $(\mathrm{Su}(\mathrm{H}))$ transcription factor activating the expression of target genes (Bray and Furriols, 2001).

The best-characterized functions of this pathway in flies are related to neurogenesis (lateral inhibition), dorso-ventral boundary formation during wing imaginal disc development, longitudinal vein specification and cell fate decisions after asymmetrical cell divisions (Artavanis-Tsakonas, et al., 1999). In all these processes Notch is subject to posttranslational modifications of the extracellular domain that regulate its function. The Notch extracellular domain is characterized by 36 epidermal growth factor-like (EGF) repeats and $3 \mathrm{Lin} / \mathrm{N}$ repeats located at the $\mathrm{C}$-terminal to the EGF repeats. Genetic and biochemical analyses indicate that O-fucosyltransferase- 1 (OFUT1) and the $\beta 1,3 \mathrm{~N}$-acetylglucosaminyltransferase Fringe are able to modify particular EGF domains and change the preferential binding of Notch to Delta or Serrate ligands (Bruckner et al., 2000; Kelley et al., 1987; de Celis and Bray, 2000; Xu et al., 2007; Pei and Baker, 2008). The first step in this glycosylation cascade is the O-fucosylation catalyzed by OFUT1 at the consensus sequence $\mathrm{C}^{2} \mathrm{X}_{4-5}(\mathrm{~S} / \mathrm{T}) \mathrm{C}^{3}$, using GDP- fucose as donor sugar (Wang et al., 2001; Okajima and Irvine, 2002). Afterwards, Fringe elongates this primer moiety adding $\mathrm{N}$-acetylglucosamine (GlcNAc) in a $\beta 1,3$ linkage (Moloney et al., 2000). Drosophila cells lack the salvage pathway for GDPfucose synthesis, and therefore the only source of GDP-fucose is the de novo synthesis from GDP-mannose (Roos et al., 2002). The cytoplasmic enzyme GDP-D-mannose 4,6-dehydratase (GMD) performs the first reaction of this metabolic pathway and accordingly no GDP-fucose is detected in Gmd null mutant animals (Sasamura et al., 2007; Okajima et al., 2008).

Although 23 EGF Notch domains could be potentially glycosylated by these enzymes, only the EGF repeats 11-12 are specific sites for ligand binding (Rebay et al., 1991). Other important repeats in the regulation of Notch activity are EGF repeats 24-29, where Abruptex mutations $\left(N^{A x}\right)$ are localized (Foster, 1975; Kelley et al., 1987; de Celis et al., 1994; Brennan et al., 1997; de Celis and Bray, 2000). Delta and Serrate also contain EGF repeats that are subject to these modifications (Shao et al., 2003).

Genetic evidence indicates that Fringe is not necessary for all aspects of Notch signaling, and so far dorso-ventral boundary formation in the wing and the eye primordium are the main events dependent on Fringe activity in Drosophila (Irvine and Wieschaus, 1994; Panin et al., 1997; Dominguez and de Celis, 1998). Conversely, OFUT1 has been implicated in every process where Notch is involved (Okajima and Irvine, 2002; Sasamura et al., 2003; Stahl et al., 2008). In vivo and cell-based assays uncovered additional functions of OFUT1 related to Notch, mainly the regulation of its intracellular trafficking, endocytosis and degradation (Sasamura et al., 2007; Okajima et al., 2008). Hence, in the OFUT1 loss-of-function

\footnotetext{
${ }^{1}$ Corresponding author: alglavic@uchile.cl Center for Genomics of the Cell, Faculty of Sciences, Universidad de Chile, Casilla 653, Santiago, Chile.
} 
condition, Notch is retained in the endoplasmic reticulum, and the resulting phenotypes are reminiscent of those caused by Notch null alleles (Okajima and Irvine, 2002; Okajima et al., 2005). In contrast, OFUT1 over-expression leads to Notch degradation, probably through the endocytic-lysosome pathway, which finally produces similar phenotypic outcomes (Sasamura et al., 2007). The effects observed in the OFUT1 gain and loss-of-function conditions are not dependent on altered O-fucosylation. Thus, the expression of an OFUT1 mutant protein lacking a functional catalytic domain is sufficient to rescue the endoplasmic retention of Notch observed in Ofut $1^{-/}$cells (Okajima et al., 2008). This mutant form binds to Notch and is as effective as normal OFUT1 in the induction of Notch degradation (Okajima et al., 2005; Sasamura et al., 2007). Consequently, the expression of OFUT1 in a Gmd mutant background, therefore in cells depleted of GDP-fucose, is enough for Notch to reach the plasma membrane, and the only phenotypes observed are those related to Fringe function (Okajima et al., 2008). Together, these observations suggest that Notch does not need to be O-fucosylated when it is involved in Fringe-independent processes.

Although Notch glycosylations have been extensively explored regarding their roles in regulating Notch-ligand interactions (Shao et al., 2003; Xu et al., 2005; Xu et al., 2007) some intriguing observations require attention. First, so far the focus of O-fucosylation has been centered on Notch EGF repeats, even though they are also present in other proteins (i.e. Delta). Second, an additional O-fucosyltransferase enzyme (OFUT2) has been predicted in the Drosophila genome that could also function in these glycosylation processes (CG14789; Roos et al., 2002) and third, while Notch signaling is impaired in $G m d^{1}$ mutant animals, a clear non-autonomous rescue is detected in mosaic tissues (Okajima et al., 2008). This behavior has been tentatively explained by the proposal that nucleotidesugars (i.e. GDP-fucose) move from cell to cell through gap junctions. However, this has not been proven yet. Altogether these observations prompted us to reevaluate the role of fucose glycosylation using genetic approaches. Our results indicate that correct GMD levels specifically modulate and are essential for the stability of wild type Notch, as well as for Abruptex mutant proteins. In both cases this function is absolutely dependent on OFUT1 activity.

\section{MATERIALS AND METHODS}

Drosophila melanogaster strains and phenotypic analysis

In a mutagenesis screening designed to identify genes affecting vein differentiation, we isolated one P-GS insertion (Toba et al., 1999) 14 bp upstream from the CG8890 ( $G m d$ ) transcription starting site (EP-800) (Fig. 1B; Molnar et al., 2006). We used the following Drosophila UAS lines: UAS-N $N^{85.1}$, UAS-N $N^{2 T 5}$, UASOfut1, UAS-iOfut1 and the Gal4 lines pnr-Gal4, dll-Gal4, nubGal4, sal ${ }^{E P v_{-}}$Gal4 (sal-Gal4 or salPE, Cruz et al. 2009), 638-Gal4 and 253-Gal4 (Calleja et al., 1996; de Celis et al., 1999; Barrio and de Celis, 2004) and the P-lacZ reporter lines $N^{\text {lac Z }}$ and $E(s p l)$ $m b$ (de Celis et al., 1998). We also used the $\mathrm{N}$ Abruptex alleles $A x^{16172}$ and $A x^{M 1}$. Gmd deficient wing discs were obtained from $G m d^{1}, G m d^{H 78}$ homozygous and $G m d^{1} / G m d^{H 78}$ heteroallelic mutant larva. Medium and large Gmd clones or mosaic nota were generated in the following genetic backgrounds using the Minute technique: hsFLP1.22; Gmd ${ }^{1}$ FRT40/ M(2)z arm-lacZ
FRT 40 and l(2)cl-L3 FRT40A/Gmd ${ }^{1}$ FRT40A; UAS-FLP/pnr-Gal4, respectively. The sal-Gal4 UAS-Gmdi/SM6a and sal-Gal4 EP-800/ SM6 $a$ were obtained by meiotic recombination. All stocks not described in the text can be found in Flybase (http://www. flybase.net/). All phenotypes were analyzed at $25^{\circ} \mathrm{C}$, unless otherwise stated, and flies were mounted for microscopic examination in lactic acid-ethanol (1:1). Pictures were taken in an Axiophot microscope with a Spot digital camera and processed using Adobe Photoshop.

Generation of Gmd RNAi construct

The Gmdi DNA fragment was amplified from the RE50314 clone using the following primers: forward 5'-CCCGGCTAATTGCAATGTCG-3' and reverse 5'-GAATTCGCGCACACTGTGCG-3'. The PCR product was cloned in the pST Blue vector (Novagene) and sequenced. It was subcloned using the SacI and BamHI sites in the pHIBS vector (Nagel et al., 2002). Later the NotI/PstI fragment from the pST Blue Gmdi and PstI/XhoI fragment from the pHIBS Gmdi vector were directionally cloned in the pBK SK using the NotI and XhoI sites to obtain the hairpin construct. Finally the Gmdi construct was introduced in the pUAST vector using the KpnI and NotI sites.

\section{RT-PCR analysis}

Actin and Gmd transcriptional profiles were analysed in third instar control (da-Gal4/+) and Gmdi-expressing larvae (Gmdi/+; da-Gal4/+). 10 larvae of each genotype were collected, total RNA extracted using Trizol reagent (Invitrogene) and cDNA synthesized using the ImProm-II kit (Promega) following manufacturer instructions. PCR analysis was performed using the following protocol: denaturation $=95^{\circ} \mathrm{C} 5 \mathrm{~min}$; 27 cycles $=95^{\circ} \mathrm{C} 45 \mathrm{sec}, 55^{\circ} \mathrm{C} 1 \mathrm{~min}$. and $72^{\circ} \mathrm{C} 40 \mathrm{sec}$; final elongation $=72^{\circ} \mathrm{C} 10 \mathrm{~min}$. The following primers were used: Actin forward, 5'-GGCCGGACTCGTCGTACTCCTGC-3' and reverse, 5'-GAGCAGGAGATGGCCACCGCTGC-3'; Gmd forward, 5'-CCCGGCTAATTGCAATGTCG-3' and reverse, 5'-GAATTCGCGCACACTGTGCG-3' .

Drosophila immunohistochemistry and in situ hybridization

We used mouse monoclonals anti-Necd, anti-Nintra, anti-Sc and anti-Dl (Hybridoma bank), rabbit anti-Sal (dilution 1:200; Barrio et al., 1999), anti-senseless (dilution 1:100; a gift from Hugo Bellen) anti-Scrb (dilution 1:200) and anti-bgal (dilution 1:200; Cappel). Secondary antibodies were from Jackson Immunological Laboratories (used at 1/200 dilution). Third instar imaginal discs were dissected, fixed and stained as described in de Celis (1997). Confocal images were captured using a BioRad and LSM 510 Zeiss confocal microscopes. In situ hybridization of imaginal discs was carried out as described in de Celis (1997). Sense and anti-sense digoxigenin-labeled RNA probes were prepared with T3 and T7 RNA polymerase using the RE50314 clone as template.

\section{RESULTS}

To understand the role of fucose modifications in Drosophila development and in Notch signaling in particular, we analyzed the consequences of modifying the levels of GMD, 
the first enzyme in the de novo biosynthesis of GDP-fucose (Roos et al., 2002). We reasoned that changing locally the levels of GMD should result in variations in the levels of GDP-fucose in mutant cells. Accordingly, this might uncover the requirements of GDP-fucose during development, as well as in Notch synthesis and signaling, irrespective of the O-fucosyltransferase enzymes involved in the transfer of this sugar (OFUT1 or OFUT2) to their potential substrates.

Over-expression of GMD results in phenotypes similar to Notch loss-offunction

Gmd (CG8890) is located in the left arm of the second chromosome in the cytological position 25B5. Gene annotation (http: / / www.flybase.org/ reports / FBgn0031661.html) predicts a single transcript of $1629 \mathrm{nt}$ and a corresponding polypeptide
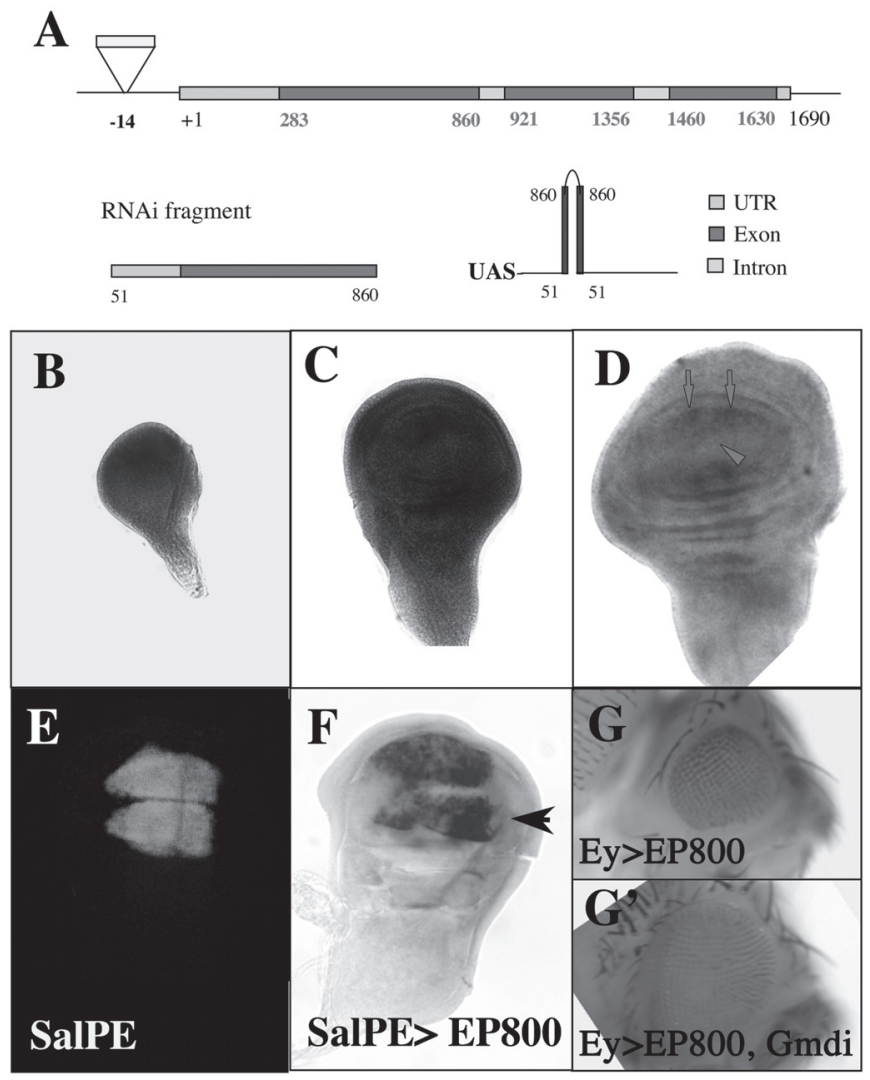

Figure 1. Gmd locus structure, expression pattern and mosaic analysis. (A) Representation of the Gmd locus indicating the position of the P-GS insertion $E P-800$ at $-14 \mathrm{bp}$ and the region used to generate the Gmdi construct. (B-D and F) In situ hybridization against $G m d$. (B-D) $G m d$ is expressed ubiquitously in wing imaginal discs from early third larva stage (B), afterward the expression is higher in the $\mathrm{L} 3$ and $\mathrm{L} 4$ presumptive regions (arrows) and lower at the dorso-ventral boundary (arrow). (E) GFP expression indicating the wing disc region that expresses the Gal4 protein in the salEPV $-G a l 4$ (SalPE) line (sal-Gal4/UAS-GFP). (F) Over-expression of $G m d$ induced by the $E P$-800 line (arrowhead, sal-Gal4/EP-800). (G) Eye adult phenotype produced by $G m d$ over-expression (ey-Gal4/EP-800). (G') The eye phenotype is reverted co-expressing the Gmdi construct (ey-Gal4, EP-800/UASGmdi). of 395aa (Fig. 1A). Protein sequence comparison showed that GMD is highly conserved among different organisms, with amino acid identities of about $70 \%$ with its human and other invertebrate counterparts (data not shown). In situ hybridization analysis revealed that Gmd is expressed ubiquitously in the wing disc throughout larval development (Fig. 1B-D), although in third instar discs its expression is higher in the L3 and L4 provein territories, and appears reduced in the region corresponding to the future wing margin (arrows and arrowhead in Fig. 1D).

P-GS insertions allow the ectopic expression of downstream genes based on the Gal4/UAS binary system (Brand and Perrimon, 1993; Toba et al., 1999). We identified one of these insertions, the EP-800 line (Molnar et al., 2006), inserted $14 \mathrm{bp}$ upstream of the Gmd transcription start site. The combination of EP-800 with a wing pouch specific driver (salPE-Gal4) induces $G m d$ expression at higher levels in this domain (Fig. 1E and F).

Analysis of adult phenotypes revealed that ectopic Gmd over-expression in the antenna-eye imaginal disc using the eyeless driver (EP-800/ey-Gal4) results in phenotypes reminiscent of Notch loss-of-function situations. Thus, over-expression of $G m d$ in this combination resulted in a reduction of the eye size and in ommatidial disorganization (Fig. 1G). As expected, the co-expression of an interference RNA directed against Gmd (Gmdi) cancelled the phenotypes generated by Gmd over-expression in this territory (Fig. 1G and $G^{\prime}$ ), indicating that 1) Gmdi specifically and efficiently targets the Gmd message and 2) the eye phenotype observed in the EP-800/ey-Gal4 combination is in fact due to GMD over-expression. The ectopic expression of Gmd in the wing blade using two Gal4 lines expressed at different levels causes phenotypes ranging from strong reductions of wing size ( $E P$ 800/nub-Gal4; Fig. 2A and C) to wing notches (638-Gal4/+; EP-800/+; Fig. 2D and F). The expression of a dominantnegative form of Notch, consisting of its trans-membrane and extracellular domains, with these same Gal4 drivers produced very similar adult phenotypes (Fig. $2 \mathrm{~B}$ and $\mathrm{E}$ ). The expression of higher than normal levels of GMD in the central wing pouch domain using the salPE-Gal4 driver (Fig. 2I) reduces the size of the wing and alters the pattern of longitudinal veins (Fig. 2G). These alterations were efficiently rescued by the co-expression of a weak activated form of Notch (Fig. 2H), indicating that the increase in GMD function somehow interferes with Notch activation. Although we have not monitored the levels of GDPfucose in these combinations, these results suggest that correct Notch signaling requires appropriate amounts of GMD and, possibly, of GDP-fucose.

To further confirm that GMD over-expression interferes with Notch signaling, we monitored the expression of Enhancer of split-mb and wingless, two Notch target genes, in the wing disc of these experimental animals. Over-expression of GMD in the wing pouch (sal-Gal4/EP-800) interrupts the expression of wingless along the dorso-ventral boundary and reduces the expression of a $E(s p l) m b$ reporter (Fig. 2J, K for $w g$ and L, $\mathrm{M}$ for $E(s p l) m b)$. In addition, GMD over-expression induces ectopic wingless expression in cells dispersed throughout the wing blade (Fig. 2K), as well as considerable cell death (data not shown). It is likely that this ectopic $\mathrm{Wg}$ signal is the consequence of the cell death induced by the high level of GMD expression (data not shown, Ryoo et al, 2004; PerezGarijo et al, 2004). 
Knockdown of Gmd reduces Notch signaling

The elimination of Gmd function in homozygous null mutant larvae $\left(G m d^{H 78}\right.$ or $\left.G m d^{1}\right)$ inhibits the induction of $w g$ expression along the dorso-ventral boundary suggesting a direct requirement of this gene for Notch activity in this region (Fig. 3A and Okajima et al., 2005). We also confirmed that Gmd mutant cells display a considerable non-autonomous behavior, as the defects in wg expression are only detected when a large fraction of the wing disc is occupied by Gmd mutant cells (arrowhead, Fig. 3B and C). To further investigate the requirement of Gmd during development and in Notch signaling we generated transgenic flies that express RNA interference against Gmd (Gmdi) under the control of UAS sequences. These experiments allow us to study the consequences of changing the temporal and spatial accumulation of GMD in the developing wing disc.

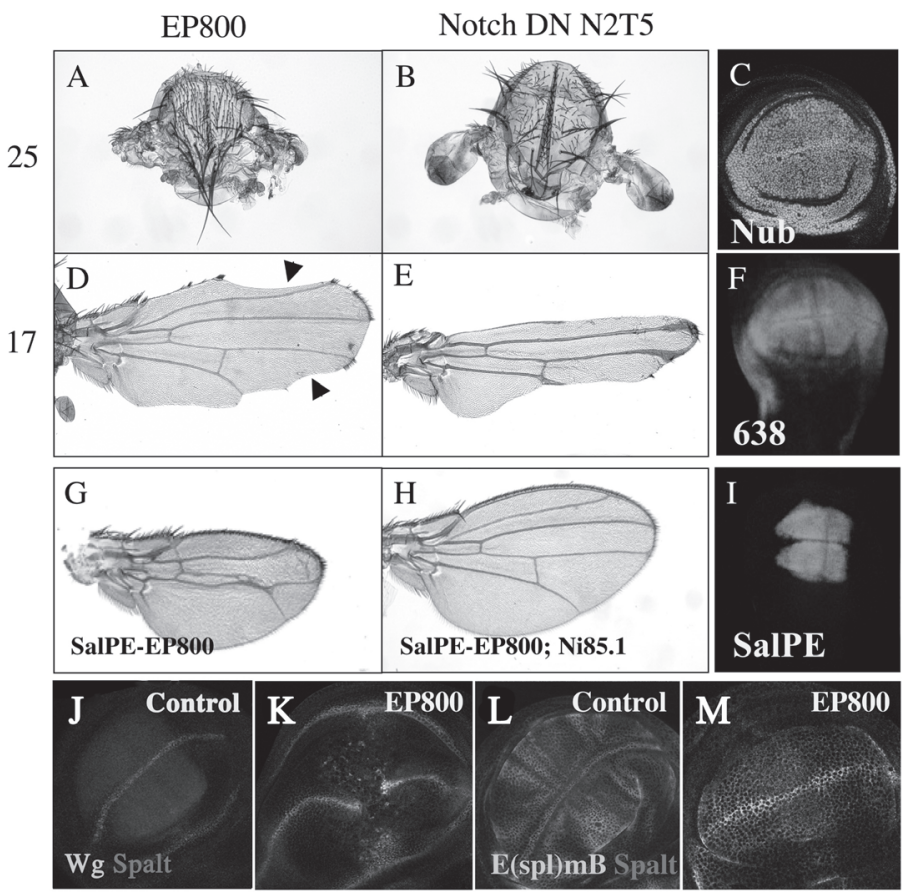

Figure 2. GMD gain-of-function produces phenotypes similar to inhibitions of Notch pathway. (A, B) Adult wing phenotype produced by the over-expression of GMD ( $A$, nub-Gal4/EP-800) or a dominant negative form of Notch (B, nub-Gal4/UAS-N ${ }^{2 T 5}$ ) using the nub-Gal4 driver at $25^{\circ} \mathrm{C}$ (C, nub-Gal4/UAS-GFP). (D, E) Adult wings obtained by GMD over-expression (D, 638-Gal4/ $E P-800)$ or $\mathrm{N}^{2 T 5}\left(\mathrm{E}, 638-G a / 4 / U A S-N^{2 T 5}\right)$ at $17^{\circ} \mathrm{C}$ in the entire wing blade (F, 638-Gal4/UAS-GFP). Note the notches at the wing margin (arrowhead in D). (G) Adult wing over-expressing GMD in the Spalt region (sal-Gal4 EP-800/+). (H) Co-expression of GMD with a weak constitutively active Notch, $\mathrm{N}^{85.1}$ (sal-Gal4 EP-800/ UAS- $N^{85.1}$ ) in the Spalt domain (I, sal-Gal4/UAS-GFP) reverts the phenotype. $(\mathrm{J}, \mathrm{K})$ Expression of $\mathrm{Wg}$ (green) and Spalt (red) in wing imaginal discs. The typical expression of $\mathrm{Wg}$ at the dorso-ventral boundary $(J)$ is disrupted in the GMD over-expression condition $(K$, sal-Gal4 EP-800/+). (L, M) Similarly, the expression of the $E(s p l)$ $m b$ reporter ( $L$, green) is reduced in this condition (M, sal-Gal4 $E P-800 /+; E(s p l) m b-c d c 2 /+)$.
RT-PCR analysis showed that expression of Gmdi efficiently reduces Gmd message (Fig. $3 \mathrm{~F}$ ) and its induction in different tissues produced adult phenotypes analogous to those caused by reductions in Notch signaling. Thus, the loss of Gmd in the wing blade (638-Gal4; UAS-Gmdi/+ or sal-Gal4/UAS-Gmdi) or in the distal leg (dll-Gal4/UAS-Gmdi) affected vein width and tarsal joint formation, respectively (Fig. $3 \mathrm{H}$, J and M). Notch signaling is also involved in the formation of the sensory organs in the thorax, affecting both the number of sensory organ precursors (SOP) and its differentiation (Schweisguth, 1995). Expression of Gmdi in the thorax, using the apterous Gal4 driver (ap-Gal4/UAS-Gmdi), prevented the formation of most micro and macrochaetae, and the remaining bristles appeared duplicated or with two shafts and no sockets (Fig. $3 \mathrm{~K})$. This phenotype was observed when the expression of Gmdi was driven in proneural clusters as well (253-Gal4/UASGmdi, data not shown), and it is likely related to the known requirement of Notch in cell-fate assignation and asymmetric cell division during sensory organ formation (Guo et al., 1996). Accordingly, in a Gmd mutant background $\left(G m d^{1} / G m d^{H 78}\right)$, Scute accumulates at higher than normal levels in proneural clusters and several SOP were selected from each cluster (arrows in Fig. 3D). This effect is also detected in the adult, where additional sensory organs appeared in several positions of the thorax (arrows in Fig. 3E). The establishment of the wing margin was only affected when Gmdi was expressed at higher levels by increasing the number of UAS-Gmdi copies (638-Gal4; UAS-Gmdi/UAS-Gmdi; Fig. 3P) or when its expression was induced in flies heterozygous for a deficiency encompassing the Gmd locus (638-Gal4; UAS-Gmdi/Df(2L)sc19-4, BL-3813; Fig. $3 \mathrm{~N})$. In these conditions the wing margin develops severe notching and the bristles show shaft duplications (Fig. $3 \mathrm{~N}$ and inset in P).

The loss of Gmd in the central wing pouch region (sal-Gal4/ UAS-Gmdi) also interfered with Notch-target gene expression, reducing $w g$ expression and $E(s p l) m b$ reporter activity in this domain (Fig. 3I, L, O and R). To confirm the impairment in Notch signaling detected upon Gmd knockdown we coexpressed Gmdi with an activated form of Notch ( $N^{\text {intra }}$ ) in the wing blade territory using the 638-Gal4 driver. In this situation (638-Gal4; UAS-Gmdi/UAS-N ${ }^{85.1}$ ) the phenotype produced by Gmdi was almost completely reversed (Fig. 3Q), further implicating Gmd function in events upstream of Notch activation. Interestingly, the reduction of GMD levels impaired both Fringe-dependent (tarsal joint and wing margin formation) and Fringe-independent processes (sensory organ formation and vein thickness). Since Fringe modulates Notch signaling through O-linked fucose modification of Notch EGF repeats, the global requirement of GMD function implies a role for GDP-fucose synthesis independent of Fringe activity. In summary, the reduction in GMD levels specifically modifies Notch signaling and it does so interfering with every aspect of Notch function in a similar way as reductions of the fucosyltransferase OFUT1 (Okajima and Irvine 2002; Sasamura et al., 2003).

\section{A GMD-dependent process stabilizes the Notch protein}

The fact that the phenotypes caused by knocking down Gmd expression were suppressed by an activated version of Notch suggests that GDP-fucose is required either for receptor activation or for its synthesis. Previously, it has been 
shown that decreasing the levels of OFUT1 influences Notch trafficking and subcellular localization, causing its retention and accumulation in the endoplasmic reticulum (Okajima et al., 2005). To identify whether Gmd reductions cause similar
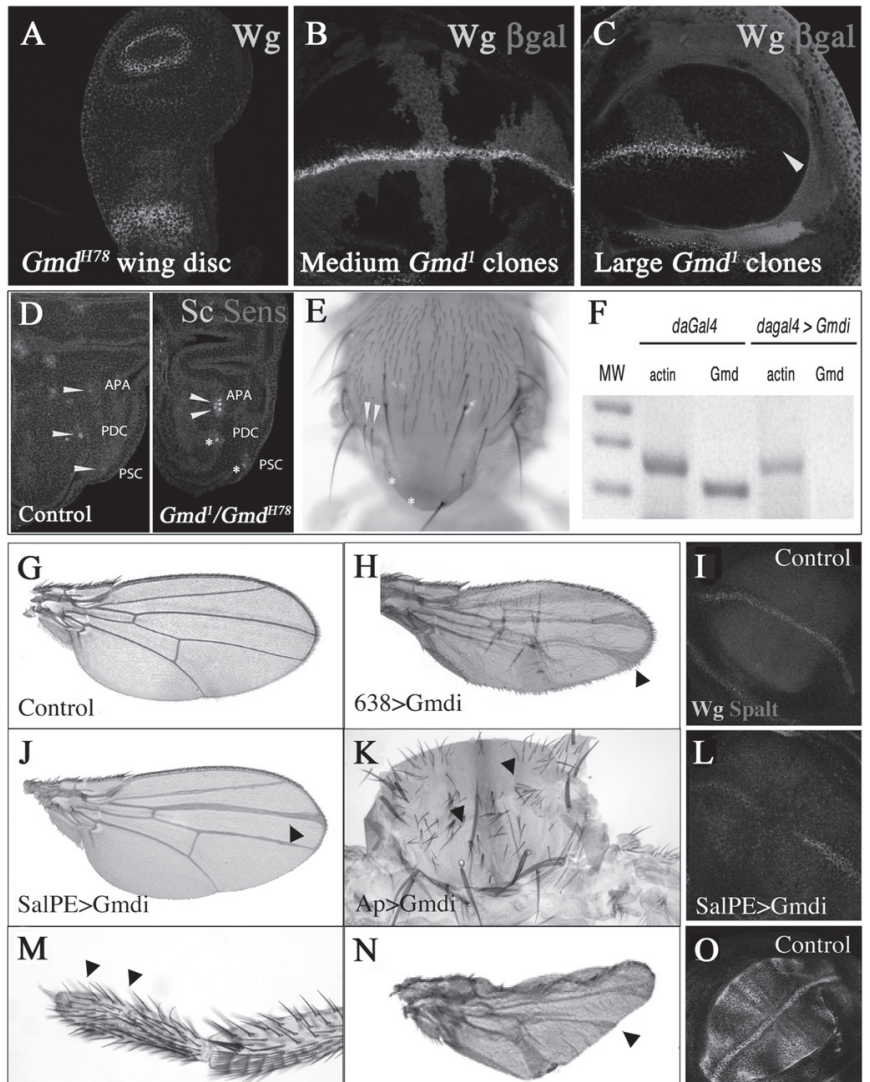

Dll>Gmdi 638>Gmdi/Df(2L)sc19-4

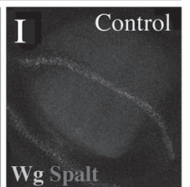

J
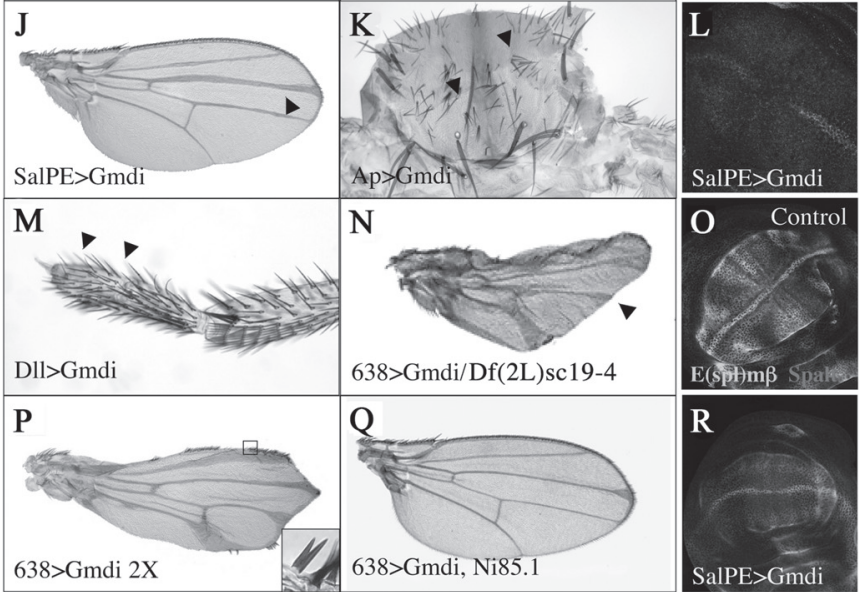

Figure 3. Gmd knockdown generates Fringe-dependent and independent Notch phenotypes. (A, B, C) Wg expression (green) in $G^{\prime} d^{1}$ homozygous mutant wing disc (A) disc and in two different wing discs with $\mathrm{Gmd}^{l}$ clones (labeled by the absence of $\beta \mathrm{Gal}$ in $\mathrm{B}$ and $\mathrm{C}$ ). Expression of $\mathrm{Wg}$ at the dorso-ventral boundary is rescued by surrounding wild type cells ( $B$, marked by $\beta$ gal expression, red) and only in very large mutant clones the loss of $G^{\prime} d^{1}$ eliminates $\mathrm{Wg}$ expression (arrowhead in C). (D) Senseless and Scute expression in the notum region of wing imaginal discs from control (left) and $G m d^{1} / G m d^{H 78}$ (right) mutant larva. Anterior postalar (APA), posterior dorsocentral (PDC) and posterior scutelar (PSC) proneural cluster are indicated (arrows). Note the presence of extra SOPs in the Gmd proneural clusters. (E) Dorsal thorax of a regional mosaic Gmd mutant animal. Mitotic recombination was induced expressing FLP in the notum with the Pannier-Gal4 driver (I(2)Cl-L3 FRT4OA/Gmd I FRT4OA; UAS-FLP/Pnr-Gal4). Note the reduction in bristle number and the appearance of an extra macrochaetae in the APA domain (arrowhead). (F) RT-PCR analysis of control $(+/+$; da-Gal4/+) and Gmdi-expressing third instar larvae (UAS-Gmdi; da-Gal4/+). (G, H, J, K, M, N, P, Q) Adult phenotypes. ( $\mathrm{I}, \mathrm{L}, \mathrm{O}, \mathrm{R})$ Confocal images of wing discs of third changes in Notch trafficking, we monitored the localization of Notch in wing discs where Gmd expression was either reduced (sal-Gal4/UAS-Gmdi) or increased (sal-Gal4/EP-800) in the central wing pouch region. Surprisingly, we found that reducing GMD expression strongly diminished the levels of Notch at the apical membrane in this territory (Fig. 4A-D). This reduction was not the consequence of a failure of the antibody to detect unmodified Notch proteins, because it was also observed using antibodies that recognize both the extracellular (Fig. 4B) as well as the Notch intracellular domain (Fig. 4C). Furthermore, the reduction in Notch protein was independent of the transcription of the Notch gene, since the reduction of Gmd does not alter the expression of a Notch reporter consisting in a P-lacZ insertion in Notch that reveals Notch transcription (Supplementary Fig. S1). Finally, the effects produced by the reduction of GMD in Notch levels seem to be specific to this receptor, because other proteins with EGF motifs susceptible to be O-fucosylated, such as Delta or Crumbs, are detected at normal levels and with a normal distribution in discs with reduced Gmd levels (Supplementary Fig. S1 and data not shown).

In order to establish whether Notch was accumulating in a subcellular compartment distinct from the apical membrane, we analyzed the expression of Notch and the apical marker Scribble in confocal transverse sections of wing discs expressing the Gmdi construct in the central wing pouch region (sal-Gal4/UAS-Gmdi). In these sections, we failed to detect Notch accumulation in any subcellular compartment, and only a very weak signal was detected at the apical cell membranes (Fig. 4D). The failure to detect Notch was not the result of a general disturbance in the apico-basal polarity of the cell, as other proteins, like Scribble, Delta, Discs large and Crumbs were normally localized at apical regions of Gmdi-expressing cells (Fig. 4D and data not shown). Therefore, we suggest that the normal processing of Notch during its transport to the apical cell membrane fails when the level of GMD is lowered, or alternatively, that Notch endocytosis and its subsequent

larva stage. (G) Control wild type wing. (H) Expression of Gmdi in the wing blade produces wing margin defects and vein thickening (arrowhead, 638-Gal4/+; UAS-Gmdi/+). (J) Expression of Gmdi in the spalt domain produces vein thickening (sal-Gal4/UAS-Gmdi). (K) Gmdi expression in the thorax inhibits bristles formation and generates shaft duplications (arrows). (M) Gmdi impairs joints formation in the distal leg (arrows, dll-Gal4/UAS-Gmdi). (N) Stronger notching and vein thickening is obtained when Gmdi is expressed in a Gmd mutant background (arrowhead, 638Gal4/+; UAS-Gmdi/Df(2L)sc19-4). (P) A similar wing phenotype is produced increasing the amount of Gmdi expressed (638-Gal4/+; UAS-Gmdi/UAS-Gmdi). In these cases shaft duplications are clearly observed in the sensory organs of the wing margin (inset). (Q) Co-expression of Gmdi with the N intra construct, N85.1, fully reverts the margin and vein thickening phenotypes caused by loss of Gmd (638-Gal4; UAS-Gmdi/UAS-N ${ }^{85.1}$ ). (I, L) Expression of Wg at the dorso-ventral boundary (green) is lost in the distal wing of sal-Gal4, UAS-Gmdi (L) compared with control disc (I). $(\mathrm{O}, \mathrm{R})$ the expression of the Notch-activity reporter $E(s p l) m b-c d c 2$ (green in $\mathrm{O}$ and $\mathrm{R}$ ) is reduced by Gmdi in sal-Gal4, UAS-Gmdi/E(spl)mbcdc2 discs (R) compared to wild type controls $(\mathrm{O})$. 
degradation are augmented under these conditions. Ectopic expression of GMD also affected Notch localization (Fig. 4E). However, in these discs apico-basal polarity was severely disturbed and the epithelial integrity is lost as evidenced by the extrusion of dying cells. These observations suggest a more general effect of GMD excess in protein trafficking that could be caused by lowering the amount of GDP-L-mannose, the substrate of GMD, which plays key roles in the secretory pathway.

\section{An OFUT1/GDP-fucose sequential mechanism controls Notch stability}

OFUT1 activity is required for Notch protein trafficking from the ER to the cell membrane. To perform this function, the O-fucosyltransferase activity of OFUT1 is not essential (Okajima et al., 2008), and therefore it has been proposed that OFUT1 acts as a chaperone that prevents inappropriate folding
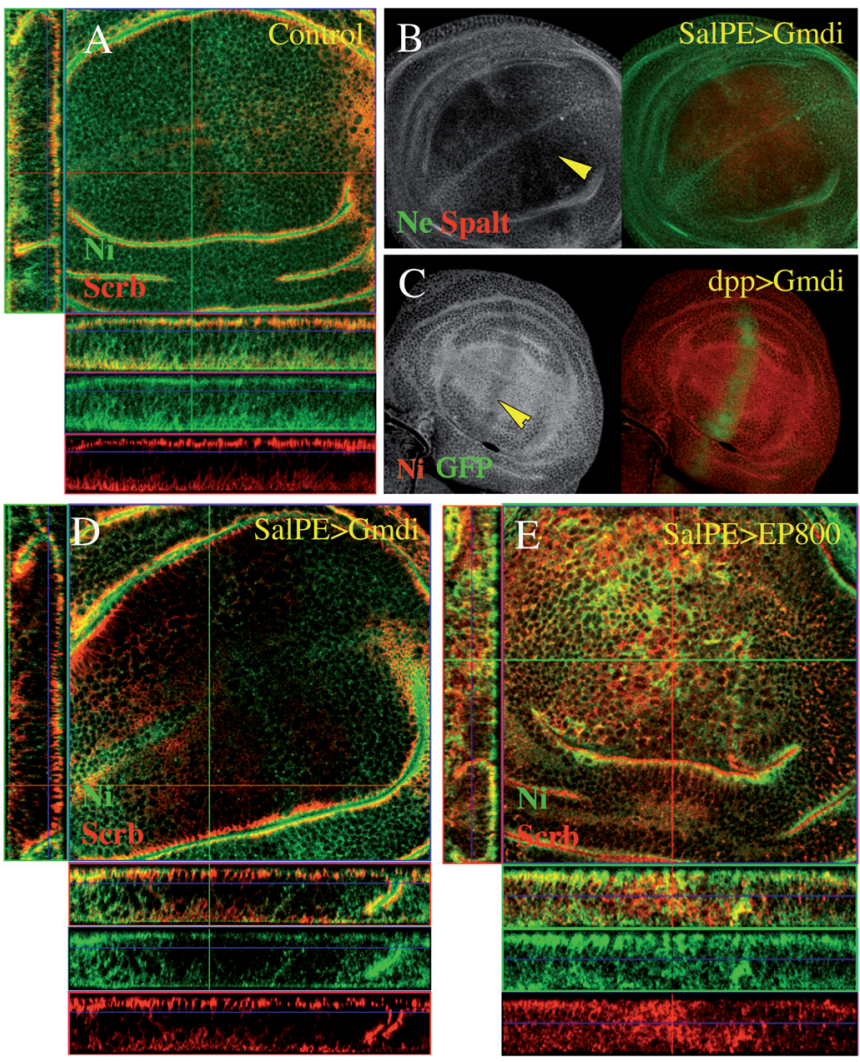

Figure 4. Expression of Gmdi reduce Notch protein levels. (A) Confocal image and the corresponding $\mathrm{Z}$ section of a control third instar wing disc stained for Notch (green) and Scribble (red) proteins. Both proteins localize preferentially at the membrane apical region. $(B, C)$ Expression of Gmdi in the central wing disc region ( $\mathrm{B}$, sal-Gal4, UAS-Gmdi) or in a central stripe (dpp-Gal4 UAS-GFP/UAS-Gmdi) decreases the amount of Notch protein detected using two different antibodies directed against the extracellular domain ( $\mathrm{Ne}$, green in $\mathrm{B}$ ) or the intracellular domain (Ni, red in C). (D) Notch (green) is not accumulated in intracellular compartments in cell expressing the Gmdi construct. The localization of Scribble (red) is not altered in this condition (salGal4, UAS-Gmdi). (E) Over-expression of GMD in the central wing disc region (sal-Gal4/EP-800) disrupts the epithelial structure. of Notch (Okajima et al., 2005). Thus, OFUT1 reduction leads to Notch accumulation at the ER, blocking its processing and apical localization and, in consequence, preventing its potential activation (Okajima et al. 2005). In agreement with previous results, expression of an Ofut1 interference RNA (iOfut1) in the central wing pouch domain (sal-Gal4/UAS-iOfut1) increases Notch accumulation in intracellular domains, causing a reduction in Notch activity and consequently Notch loss-offunction phenotypes in the adult wing (arrows Fig. 5A, B and B'; Okajima and Irvine, 2002). Likewise, loss of GMD function also reduces Notch signaling and causes a vein thickening phenotype, although in this case the defect is caused by a decrease in Notch protein levels (arrows Fig. 5C, D and D'). Co-expression of the two RNAi constructs, iOfut1 and Gmdi, also resulted in adult wing phenotypes compatible with reductions of Notch signaling (Fig. 5E), but notably, in this case Notch protein accumulated as in iOfut1-expressing discs (compare Fig. 5F-F' with B-B'). These results suggest that the GMD-dependent process related to Notch stability occurs after the traffic of Notch from the ER to the Golgi apparatus and that OFUT1 is essential for this route of Notch degradation. These observations indicate that an unbalance of GDP-fucose in the cell facilitates Notch degradation, likely through an endocyticlysosome pathway assisted by OFUT1, further supporting the notion that OFUT1, acting from the extracellular milieu, directs the endocytosis and degradation of Notch (Sasamura et al., 2007).
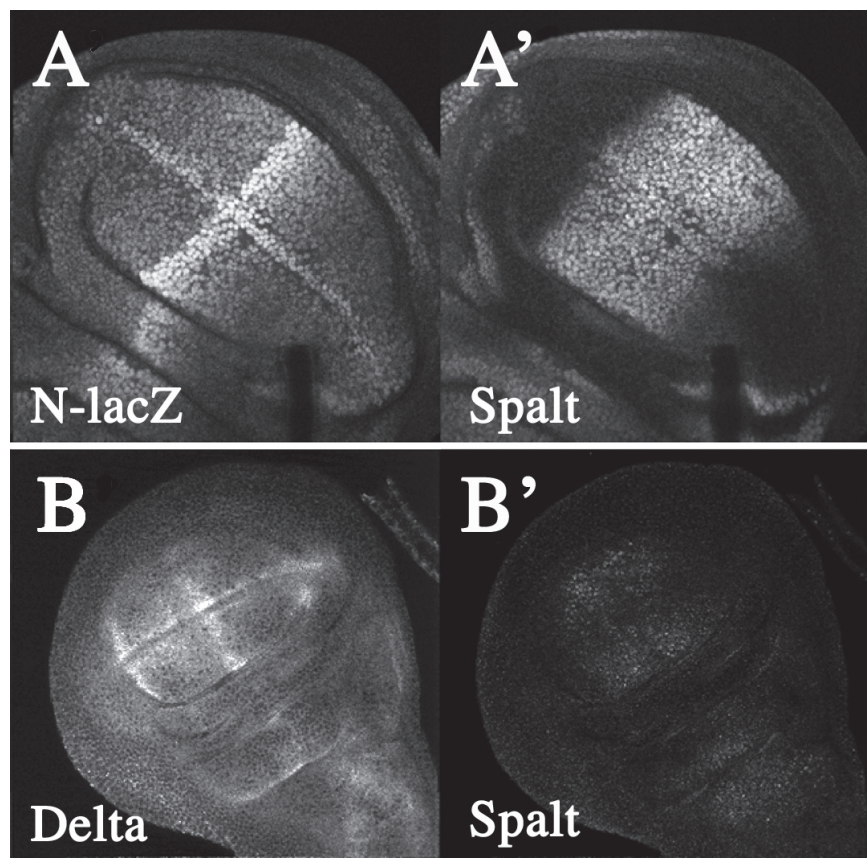

Supplementary Figure S1. The reduction of GMD does not affect Notch transcription or Delta expression. ( $\left.A, A^{\prime}, A^{\prime \prime}\right)$ Expression of Gmdi in the Spalt domain (Spalt, red) does not alter the expression of the Notch P-lacZ insertion reporter ( $N^{\text {lacZ }}$; green). $\left(B, B^{\prime}, B^{\prime \prime}\right)$ Delta (green) localization and stability are not modified by the reduction of GMD in the central wing imaginal disc region (Spalt, red; sal-Gal4 UAS-Gmdi/+). 


\section{Abruptex alleles are subject to OFUT1 and GMD-dependent control}

To define the EGF domains involved in the GMD- and OFUT1-dependent mechanism that controls Notch levels, we analyzed the effect of changing the amount these proteins in Abruptex mutant backgrounds. Abruptex alleles are caused by point mutations within EGF repeats 24 to 29 of the Notch extracellular domain and modify Notch activation in a liganddependent manner (Kelley et al., 1987; de Celis et al., 1994; Brennan et al., 1997; de Celis and Bray, 2000). In addition, in vitro binding assays showed that the Abruptex region specifically interacts with the ligand binding region of Notch, and this has been proposed to serve as a barrier for the ligandinduced activation of Notch (Pei and Baker, 2008).

$A x^{16172}$ viable hemizygotes show higher levels of Notch signaling, specifically affecting wing growth and vein development (Fig. 6A). Gmdi expression using the nubbin-Gal4 driver in the wing blade region of $A x^{16172}$ ( $A x^{16172}$; nub-Gal4/ UAS-Gmdi), as well as in $A x^{M 1}$ background (data not shown), completely suppressed the $A x$ gain-of-function phenotype. Moreover, the veins appeared thickened, suggesting that reductions in GMD function produce a Notch deficiency even in the presence of hyper-activable Notch proteins (Fig. 6B). As

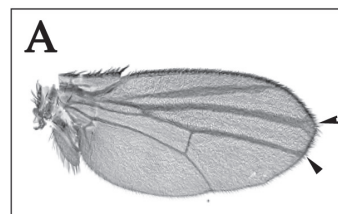

SalPE $>$ iOfut

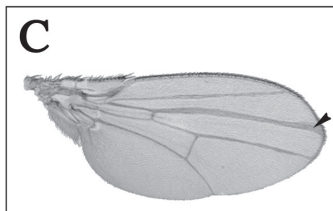

SalPE $>$ Gmdi
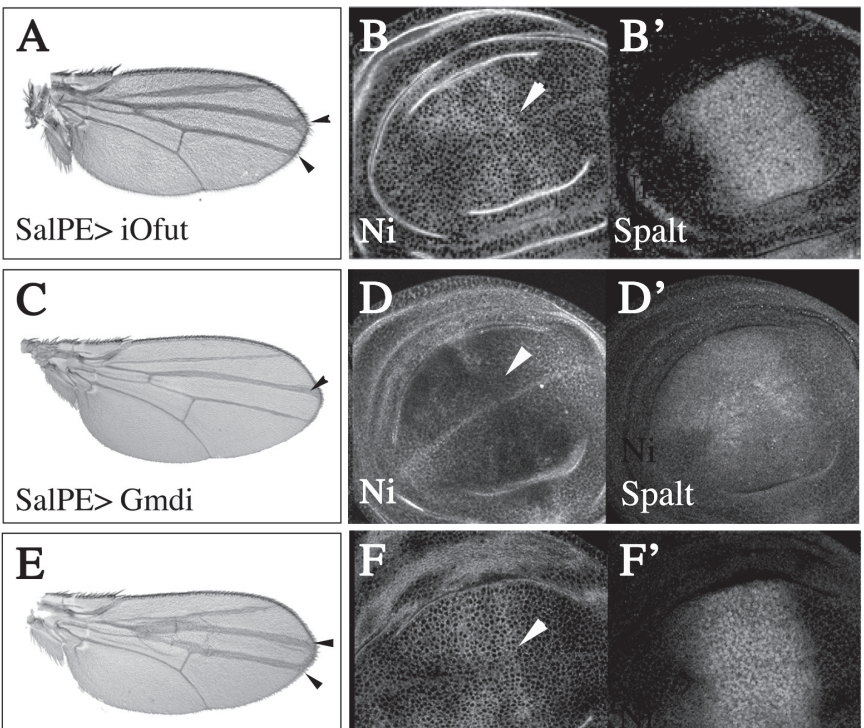

SalPE $>$ iOfut, Gmdi
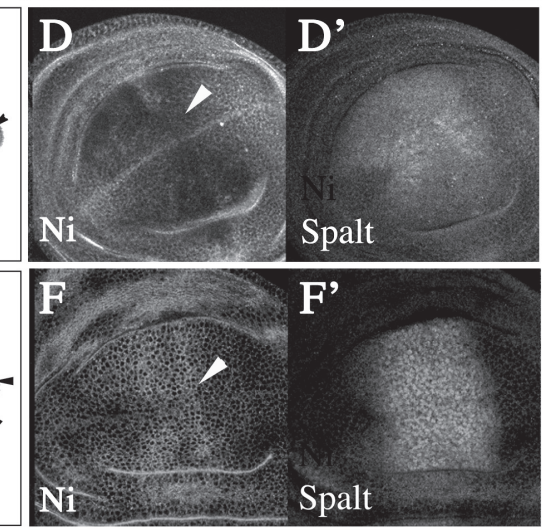

Figure 5. Notch destabilization in GMD-deficient cells is dependent on OFUT1 protein. (A) Adult wing knockdown for OFUT1. Expression of iOfut 1 in the central wing region produces vein thickening (arrows, UAS-iOfut1; sal-Gal4/+). (B, B') Notch is accumulated intracellularly in the domain expressing the iOfut 1 construct (arrowhead). (C) Adult wing knockdown for GMD. Expression of Gmdi in the central wing region produces vein thickening (arrows, sal-Gal4/UAS-Gmdi). (D, D') Notch is reduced in the domain of Gmdi expression (arrowhead). (E) Adult wing knockdown for OFUT1 and GMD. Expression of iOfut 1 and Gmdi in the central wing region produces similar vein thickening (arrows, UAS-iOfut1; sal-Gal4, UAS-Gmdi/+). (F, $\left.\mathrm{F}^{\prime}\right)$ Notch is accumulated intracellularly in the domain co-expressing the iOfut 1 and Gmdi constructs, indicating that Notch degradation in Gmdi expressing cells is absolutely dependent on OFUT 1 protein. in Notch wild type conditions, the levels of $A x$ mutant proteins are also decreased when Gmdi is expressed in the central wing pouch domain (A $x^{16172}$;sal-Gal4/UAS-Gmdi; Fig. 6E-F), demonstrating that the GMD-dependent control mechanism is independent of the EGF domains mutated in these $A x$ alleles. A similar reduction in Notch activity was observed knockingdown Ofut1 function in $A x$ backgrounds ( $A x^{16172}$;sal-Gal4/UASiOfut1; Fig. 6C). Conversely, the over-expression of GMD using the sal-Gal4 driver in $A x$ mutants enhances the loss of vein phenotype produced by the $A x$ allele $\left(A x^{16172}\right.$; sal-Gal4/EP800; arrows indicating L3 and L5 in Fig. 6D), and in this case the mutant Notch protein appeared at higher than normal levels and in a punctuate distribution (Fig. 6G).

\section{DISCUSSION}

Notch is a glycosylated transmembrane receptor with central roles during development (Artavanis-Tsakonas et al., 1999). The Notch protein suffers several glycosylation steps that affect Notch-ligand interactions (Xu et al., 2005; Bray, 2006;

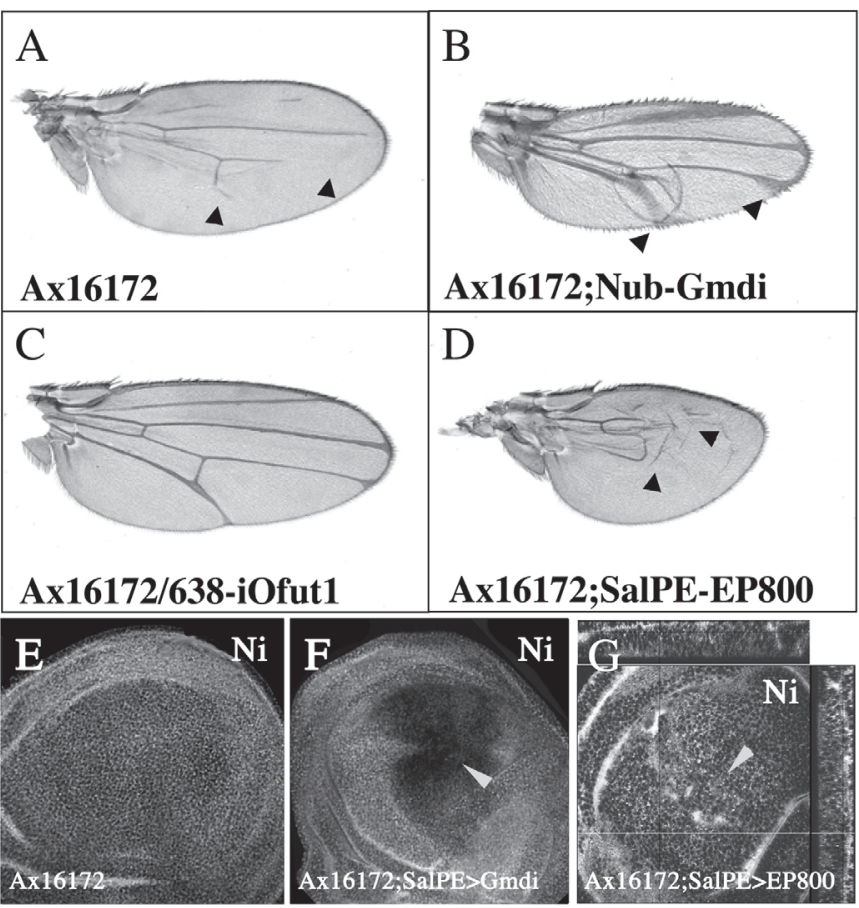

Figure 6. Abruptex alleles are subject to the OFUT1 quality control. (A) Adult wing hemizygous for the Notch Abruptex allele $A x^{16172}$. The hyperactivation of the Notch pathway partially suppresses the development of the L2, L4 and L5 veins (arrows). (B) The Abruptex phenotype is completely reverted by Gmdi and the resulting wings shows strong vein thickening (arrows, $A x^{16172}$; nub-Gal4 UAS-Gmdi/+). (C) Similar reversion is observed reducing OFUT1 levels (Ax ${ }^{16172} / 638-G a l 4$, UAS-iOfut 1$)$. (D) GMD over-expression enhances the vein phenotype produced by the $A x^{16172}$ allele (arrowhead, $A x^{16172}$; sal-Gal4 EP-800/+). (E) Notch expression in $A x^{16172}$ hemizygous wing imaginal discs. (F) Similarly to wild type Notch, $A x^{16172}$ protein is reduced in GMD-knockdown cells (arrowhead, Ax ${ }^{16172}$; sal-Gal4, UAS-Gmdi/+). (G) GMD overexpression in this mutant background increment the levels of Notch protein (arrowhead, $A x^{16172}$; sal-Gal4, EP-800/+). 
$\mathrm{Xu}$ et al., 2007). Classical sugar modifications occur in the ER and Golgi apparatus by a battery of glycosyltransferases (Helenius and Aebi, 2001). So far, two of these enzymes have been involved in Notch synthesis. The O-fucosyltransferase neurotic/Ofut1 (Sasamura et al., 2003; Okajima and Irvine, 2002), which potentially transfers fucose to the EGF domains (Panin et al., 2002; Shao et al., 2003; Xu et al., 2005) and the $\beta 1,3 \mathrm{~N}$ acetylglucosaminyltransferase Fringe, which elongates this priming moiety by adding $\mathrm{N}$-acetylglucosamine units (Lei et al., 2003; Shao et al., 2003).

A recent report has uncovered a quality control mechanism that affects Notch synthesis, which unexpectedly, relies on a non-enzymatic activity of OFUT1 (Okajima et al., 2005; Okajima et al., 2008). Besides this function, Sasamura et al. (2007) showed that OFUT1 acting from the extracellular environment was able to down regulate Notch by enhancing its endocytosis towards the degradative pathway. In addition, our results show that it is the balance of GDP-fucose and OFUT1 what modulates Notch signaling by regulating the amount of functional Notch protein at the cell surface.

The phenotypes caused by changes in GMD activity are related to loss of Notch signaling

Gmd null alleles and OFUT1 mutant forms have been used to assess the specific dependency of Notch signaling on O-fucosylation (Okajima et al., 2005; Sasamura et al., 2007; Okajima et al., 2008). This has lead to the conclusion that O-fucosylation is exclusively required for Fringe-dependent processes (Okajima et al., 2008). We found that over-expression of Gmd leads to a reduction of Notch activity. This effect is reverted by a weak constitutively active form of Notch, indicating that GMD over-expression interferes upstream of Notch activation. GMD is the enzyme that performs the first step in the synthesis of GDP-fucose in Drosophila (Roos et al., 2002). In accordance to this, no GDP-fucose is detected in Gmd null mutant embryos (Sasamura et al., 2007) and wing discs from these animals show no expression of $w g$ at the dorso-ventral boundary (Micchelli et al., 1997; Okajima and Irvine, 2002; Okajima et al., 2008). Clonal analysis has shown a non cell-autonomous requirement of $G m d$, preventing the analysis of GMD function in mosaics. By expressing the Gmd RNAi construct in large wing domains, we were able to analyze the cellular consequences of Gmd and GDP-fucose reductions. In this background there is a strong reduction of Notch protein levels that was not detected in mosaic Gmd tissues (data not shown). Remarkably, the adult phenotypes produced by lowering GMD affected both Fringe-dependent and independent processes, and these effects were completely reverted by the expression of a Notch intracellular construct, indicating that under low GDP-fucose Delta/Serrate activation of Notch is impaired.

An appropriate balance between GMD activity and OFUT1 modulates Notch stability

Our GMD knockdown conditions reduce Notch protein levels without noticeable changes in Notch gene expression, Delta levels or apico-basal polarity. Thus, appropriate levels of GDPfucose are specifically essential for Notch stability, but not for the accumulation or function of other proteins that contain EGF domains. The observation that Notch is not retained in the
ER in GMD-depleted cells additionally confirms that OFUT1 working as a chaperone, and not O-fucosylation, is necessary for Notch trafficking (Okajima et al., 2005; Okajima et al., 2008). But how is Notch destabilized under our experimental conditions? Sasamura et al. (2008) have recently identified a second role of OFUT1 in Notch signaling that might explain our observations. They showed that OFUT1 acting from the extracellular space enhances Notch endocytosis promoting its degradation (Sasamura et al., 2007). Interestingly, loss of Gmd fails to promote Notch degradation when the levels of OFUT1 are lowered, indicating that OFUT1 is required for Notch degradation in conditions of low levels of GDP-fucose. Okajima et al., (2008) showed that in Drosophila S2 cells, where OFUT1 is endogenously expressed, the treatment with a dsRNA against Gmd produces a reduction in the secretion of Notch. These observations suggest us that under acute GDP-fucose restrictions, there is an increment in the ability of OFUT1 to remove Notch from the cell surface leading to its degradation. Our in vivo analysis directly implicates the level of GDP-fucose in the ability of OFUT1 to induce the removal of Notch from the cell surface.

In our view, during Notch synthesis, OFUT1 sequentially associates and facilitates the folding of the Notch extracellular domain. The O-fucosylation performed by OFUT1 stabilizes the conformation of particular EGF domains allowing the progression of Notch through the secretory pathway (Okajima et al., 2005; Okajima et al., 2008). The association of Notch with OFUT1 decreases after O-fucosylation and, consequently, only small amounts of OFUT1 reach the plasma membrane. In this scenario, the endocytosis and degradation promoted by extracellular OFUT1 are kept at low levels (Sasamura et al., 2007). However, if O-fucosylation is not correctly executed, for instance due to the lack of GDP-fucose, higher levels of OFUT1 could progress to the plasma membrane in association with Notch, inducing its rapid endocytosis and posterior degradation. Such a mechanism might ensure that only correctly modified Notch proteins are retained in the membrane ready for ligand interactions.

\section{Which Notch domains are involved in GMD/OFUT1 stabilization?}

Several EGF repeats have been implicated in Notch-ligand binding and Fringe activity (Xu et al., 2007). One of them, the Abruptex region, has been shown to be part of intramolecular contacts involving EGF 11-12 and EGF 22-27 that competitively modulates Notch-ligand interactions (Pei and Baker, 2008). The low stability of Notch protein observed with our Gmdi construct and its retention in OFUT1-depleted cells are also observed in Abruptex backgrounds ( $A x^{16172}$ and $\left.A x^{M 1}\right)$. The suppression of the hyperactivation properties of these alleles suggests that the OFUT1-dependent mechanism for Notch downregulation proposed here and the previously reported OFUT1-assisted folding of Notch (Okajima et al., 2005) are also operative in these Notch mutants. Thus, our observations indicate that the EGF domains mutated in Abruptex alleles are not involved in the structural roles of OFUT1 during Notch trafficking. It is worth mentioning that increments in GMD levels augmented the Notch gain-of-function phenotype and protein accumulation in $A x$ background, while the same treatment resulted in a reduction of Notch activity in normal discs. The opposite responses of Ax and wild type Notch proteins to higher than normal levels of GMD suggest that a 
limiting aspect of the hyperactivity observed in Ax proteins is dependent on the presence of GDP-fucose. Thus under high GDP-fucose Ax proteins seem to be less susceptible to OFUT1induced endocytosis and degradation than wild type Notch. Alternatively, GMD over-expression could reduce the amount of GDP-L-mannose, the substrate of GMD, which is required in a control and sorting mechanism operating in the secretory pathway (Helenius and Aebi, 2001). Perhaps Ax proteins and not wild type Notch partially bypass this GDP-L-mannosedependent control mechanism resulting in the activation or blockage of Notch signaling, respectively.

\section{ACKNOWLEDGMENTS}

We would like to thank Miguel Allende for critical reading and insights during the preparation of this manuscript and Hugo Bellen, the Bloomington Stock Center and the Hybridoma Bank for fly stocks and reagents. This work was funded by ICM P06-039F grant to A.G. and by a BFU2009-09403 grant of the M.E.C. to J.F.dC. An institutional grant from the Ramón Areces Foundation to the CBMSO is also acknowledged.

\section{REFERENCES}

ARTAVANIS-TSAKONAS S, RAND MD, LAKE RJ. (1999) Notch signaling: cell fate control and signal integration in development. Science 284(5415):770-6.

BARRIO R, DE CELIS JF, BOLSHAKOV S, KAFATOS FC. (1999) Identification of regulatory regions driving the expression of the Drosophila spalt complex at different developmental stages. Dev Biol 215(1):33-47.

BARRIO R, DE CELIS JF. (2004) Regulation of spalt expression in the Drosophila wing blade in response to the Decapentaplegic signaling pathway. Proc Natl Acad Sci U S A 101(16):6021-6.

BRAND AH, PERRIMON N. (1993) Targeted gene expression as a means of altering cell fates and generating dominant phenotypes. Development 118(2):401-15.

BRAY S, FURRIOLS M. (2001) Notch pathway: making sense of suppressor of hairless. Curr Biol 11(6):R217-21.

BRAY SJ. (2006) Notch signalling: a simple pathway becomes complex. Nat Rev Mol Cell Biol 7(9):678-89.

BRENNAN K, TATESON R, LEWIS K, ARIAS AM. (1997) A functional analysis of Notch mutations in Drosophila. Genetics 147(1):177-88.

BRÜCKNER K, PEREZ L, CLAUSEN H, COHEN S. (2000) Glycosyltransferase activity of Fringe modulates Notch-Delta interactions. Nature 406(6794):411-5.

CALLEJA M, MORENO E, PELAZ S, MORATA G. (1996) Visualization of gene expression in living adult Drosophila. Science 274(5285):252-5.

DE CELIS JF, BARRIO R, KAFATOS FC. (1999) Regulation of the spalt/ spalt-related gene complex and its function during sensory organ development in the Drosophila thorax. Development 126(12):2653-62.

DE CELIS JF, BRAY SJ. (2000) The Abruptex domain of Notch regulates negative interactions between Notch, its ligands and Fringe. Development 127(6):1291-302.

DE CELIS JF, GARCIA-BELLIDO A. (1994) Modifications of the notch function by Abruptex mutations in Drosophila melanogaster. Genetics 136(1):183-94.

DE CELIS JF. (1997) Expression and function of decapentaplegic and thick veins during the differentiation of the veins in the Drosophila wing. Development 124(5):1007-18.

DE CELIS, JF, TYLER D, DE CELIS J, BRAY SJ (1998). Notch signalling mediates segmentation of the Drosophila leg. Development 125, 46174626.

DOMÍNGUEZ M, DE CELIS JF. (1998). A dorsal/ventral boundary established by Notch controls growth and polarity in the Drosophila eye. Nature 396(6708):276-8.

FOSTER GG. (1975) Negative complementation at the notch locus of Drosophila melanogaster. Genetics 81(1):99-120.

GUO M, JAN LY, JAN YN. (1996) Control of daughter cell fates during asymmetric division: interaction of Numb and Notch. Neuron 17(1):27-41.
HELENIUS A, AEBI M. (2001) Intracellular functions of N-linked glycans. Science 291(5512):2364-9.

IRVINE KD, WIESCHAUS E. (1994) fringe, a Boundary-specific signaling molecule, mediates interactions between dorsal and ventral cells during Drosophila wing development. Cell 79(4):595-606.

KELLEY MR, KIDD S, DEUTSCH WA, YOUNG MW. (1987) Mutations altering the structure of epidermal growth factor-like coding sequences at the Drosophila Notch locus. Cell 51(4):539-48.

KLEIN T, ARIAS AM. (1998) Interactions among Delta, Serrate and Fringe modulate Notch activity during Drosophila wing development. Development 125(15):2951-62.

LAI EC. (2004) Notch signaling: control of cell communication and cell fate. Development 131(5):965-73.

LEI L, XU A, PANIN VM, IRVINE KD. (2003) An O-fucose site in the ligand binding domain inhibits Notch activation. Development 130(26):6411-21.

MICCHELLI CA, RULIFSON EJ, BLAIR SS. (1997) The function and regulation of cut expression on the wing margin of Drosophila: Notch, Wingless and a dominant negative role for Delta and Serrate. Development 124(8):1485-95.

MOLNAR C, LÓPEZ-VAREA A, HERNÁNDEZ R, DE CELIS JF. (2006) A gain-of-function screen identifying genes required for vein formation in the Drosophila melanogaster wing. Genetics 174(3):1635-59.

MOLONEY DJ, PANIN VM, JOHNSTON SH, CHEN J, SHAO L, WILSON R, WANG Y, STANLEY P, IRVINE KD, HALTIWANGER RS, VOGT TF. (2000) Fringe is a glycosyltransferase that modifies Notch. Nature 406(6794):369-75

NAGEL AC, MAIER D, PREISS A. (2002) Green fluorescent protein as a convenient and versatile marker for studies on functional genomics in Drosophila. Dev Genes Evol 212(2):93-8.

NELLESEN DT, LAI EC, POSAKONY JW. (1999) Discrete enhancer elements mediate selective responsiveness of enhancer of split complex genes to common transcriptional activators. Dev Biol 213(1):33-53.

OKAJIMA T, IRVINE KD. (2002) Regulation of notch signaling by o-linked fucose. Cell 111(6):893-904.

OKAJIMA T, REDDY B, MATSUDA T, IRVINE KD. (2008) Contributions of chaperone and glycosyltransferase activities of O-fucosyltransferase 1 to Notch signaling. BMC Biol. 6:1.

OKAJIMA T, XU A, LEI L, IRVINE KD. (2005) Chaperone activity of protein O-fucosyltransferase 1 promotes notch receptor folding. Science 307(5715):1599-603.

PANIN VM, PAPAYANNOPOULOS V, WILSON R, IRVINE KD. (1997) Fringe modulates Notch-ligand interactions. Nature 387(6636):908-12.

PANIN VM, SHAO L, LEI L, MOLONEY DJ, IRVINE KD, HALTIWANGER RS. (2002) Notch ligands are substrates for protein O-fucosyltransferase-1 and Fringe. J Biol Chem 277(33):29945-52.

PEI Z, BAKER NE. (2008) Competition between Delta and the Abruptex domain of Notch. BMC Dev Biol 8:4.

PEREZ-GARIJO A, MARTIN FA, MORATA G. (2004). Caspase inhibition during apoptosis causes abnormal signalling and developmental aberrations in Drosophila. development 131, 5591-5598.

REBAY I, FLEMING RJ, FEHON RG, CHERBAS L, CHERBAS P, ARTAVANIS-TSAKONAS S. (1991) Specific EGF repeats of Notch mediate interactions with Delta and Serrate: implications for Notch as a multifunctional receptor. Cell 67(4):687-99.

RYOO HD, GORENC T, STELLER H. (2004) Apoptotic cells can induce compensatory cell proliferation through the JNK and the Wingless signaling pathways. Dev. Cell 7, 491-501.

ROOS C, KOLMER M, MATTILA P, RENKONEN R. (2002) Composition of Drosophila melanogaster proteome involved in fucosylated glycan metabolism. J Biol Chem 277(5):3168-75.

SASAMURA T, ISHIKAWA HO, SASAKI N, HIGASHI S, KANAI M, NAKAO S, AYUKAWA T, AIGAKI T, NODA K, MIYOSHI E, TANIGUCHI N, MATSUNO K. (2007) The O-fucosyltransferase O-fut1 is an extracellular component that is essential for the constitutive endocytic trafficking of Notch in Drosophila. Development 134(7):1347-56.

SASAMURA T, SASAKI N, MIYASHITA F, NAKAO S, ISHIKAWA HO, ITO M, KITAGAWA M, HARIGAYA K, SPANA E, BILDER D, PERRIMON $\mathrm{N}$, MATSUNO K. (2003) neurotic, a novel maternal neurogenic gene, encodes an O-fucosyltransferase that is essential for Notch-Delta interactions. Development 130(20):4785-95.

SCHWEISGUTH F. (1995) Suppressor of Hairless is required for signal reception during lateral inhibition in the Drosophila pupal notum. Development 121(6):1875-84.

SHAO L, MOLONEY DJ, HALTIWANGER R. (2003) Fringe modifies O-fucose on mouse Notch1 at epidermal growth factor-like repeats 
within the ligand-binding site and the Abruptex region. J Biol Chem 278(10):7775-82.

STAHL M, UEMURA K, GE C, SHI S, TASHIMA Y, STANLEY P. (2008) Roles of Pofut1 and O-fucose in mammalian Notch signaling. J Biol Chem 283(20):13638-51

TOBA G, OHSAKO T, MIYATA N, OHTSUKA T, SEONG KH, AIGAKI T.

(1999) The gene search system. A method for efficient detection and rapid molecular identification of genes in Drosophila melanogaster Genetics 151(2):725-37.
WANG Y, SHAO L, SHI S, HARRIS RJ, SPELLMAN MW, STANLEY P, HALTIWANGER RS. (2001) Modification of epidermal growth factorlike repeats with O-fucose. Molecular cloning and expression of a novel GDP-fucose protein O-fucosyltransferase. J Biol Chem 276(43):40338-45.

XU A, HAINES $\mathrm{N}$, DLUGOSZ M, RANA NA, TAKEUCHI $\mathrm{H}$, HALTIWANGER RS, IRVINE KD. (2007) In vitro reconstitution of the modulation of Drosophila Notch-ligand binding by Fringe. J Biol Chem 282(48):35153-62.

XU A, LEI L, IRVINE KD. (2005) Regions of Drosophila Notch that contribute to ligand binding and the modulatory influence of Fringe. J Biol Chem 280(34):30158-65. 